\title{
Caloric restriction reverses the deficits in leptin receptor protein and leptin signaling capacity associated with diet-induced obesity: role of leptin in the regulation of hypothalamic long-form leptin receptor expression
}

\author{
J Wilsey ${ }^{1,2}$ and P J Scarpace ${ }^{1,2}$ \\ ${ }^{1}$ Geriatric Research, Education and Clinical Center, Department of Veterans Affairs Medical Center, Gainesville, Florida 32608-1197, USA \\ ${ }^{2}$ Department of Pharmacology and Therapeutics, University of Florida College of Medicine, Gainesville, Florida 32608, USA \\ (Requests for offprints should be addressed to P J Scarpace; Email: scarpace@ufl.edu)
}

\begin{abstract}
The objectives of this study were to determine if reduced long-form leptin receptor $(\mathrm{ObRb})$ expression in dietinduced obese (DIO) animals is associated with deficits in maximal leptin signaling and, secondly, to establish the effects of short-term caloric restriction (CR) on $\mathrm{ObRb}$ expression and function. Groups of DIO and life-long chow-fed $(\mathrm{CHOW}) \mathrm{F} 344 \times$ BN male rats, aged 6 months, were given an i.c.v. injection containing $2 \mu \mathrm{g}$ leptin or artificial cerebrospinal fluid (ACSF) vehicle. Leptin induced a $>6$-fold increase in STAT3 phosphorylation in CHOW rats, but less than 2-fold increase in DIO. Reduced maximal leptin-stimulated STAT3 phosphorylation in DIO rats was coupled with a decline in both $\mathrm{ObRb}$ expression and protein. At this point, subgroups of
\end{abstract}

DIO and CHOW animals underwent CR for 30 days and were then tested for acute leptin responsiveness. CR resulted in a 45 and $85 \%$ increase respectively in leptinstimulated STAT3 phosphorylation in CHOW and DIO animals. Similarly, CR increased ObRb expression and protein in both CHOW and DIO animals. To explore the role of leptin in regulating ObRb expression, we reversibly overexpressed leptin in the hypothalamus and found that ObRb mRNA inversely follows central leptin expression. By enhancing both $\mathrm{ObRb}$ expression and signaling capacity, CR may enhance leptin responsiveness in leptinresistant DIO animals.

Journal of Endocrinology (2004) 181, 297-306

\section{Introduction}

Common human obesity is associated with hyperleptinemia and leptin resistance. Clinical trials with leptin have been disappointing due to this phenomenon of leptin resistance in the obese state (Gura 1999) and interest in leptin to treat obesity has waned. Although it is a subject of rigorous investigation, the precise mechanism of leptin resistance in obesity is unknown. While transport of leptin across the blood-brain barrier (BBB) appears to be saturated at serum leptin levels observed in obesity (Van Heek et al. 1997, Banks et al. 1999), deficient BBB transport is clearly not the only factor contributing to leptin insensitivity. Both we and others have reported convincing evidence for a central component to leptin resistance in obese animal models including obese $\mathrm{A}^{\mathrm{y}}$ mice (Halaas et al. 1997), diet-induced obese (DIO) C57BL/6J mice (El-Haschimi et al. 2000), DIO Sprague-Dawley rats (Levin \& Dunn-Meynell 2002), and aged-obese F344 $\times$ BN rats (Shek \& Scarpace 2000, Scarpace \& Tumer 2001, Scarpace et al. 2001). Recent data from our laboratory demonstrated that DIO F344 $\times \mathrm{BN}$ rats are completely unresponsive to an i.c.v. injection of recombinant adeno-associated virus-encoding-leptin (rAAVleptin) (Wilsey et al. 2003). A similar dose of rAAV-leptin administered to age-matched chow-fed (CHOW) animals causes potent anorexia sustained for at least 150 days, thermogenesis, and a near complete loss of visceral white adipose tissue (Scarpace et al. 2002, 2003, Wilsey et al. 2003).

It has been previously demonstrated that various obese animal models have reduced leptin receptor $(\mathrm{ObRb})$ expression and/or protein in the hypothalamus. This includes high fat-fed Osborne-Mendel rats (Madiehe et al. 2000), DIO C57 mice (Lin et al. 2000), and aged-obese Wistar and $\mathrm{F} 344 \times \mathrm{BN}$ rats (Scarpace et al. 2001, Fernandez-Galaz et al. 2002). Similarly, we recently demonstrated that our DIO F344 $\times$ BN rat model also exhibits reduced ObRb expression (Wilsey et al. 2003). It is unknown whether these reductions in $\mathrm{ObRb}$ expression and protein are associated with reduced maximal leptin signaling. Our objective was to determine if DIO animals 
have reduced ObRb-mediated JAK/STAT3 signaling capacity as measured by maximal leptin-induced STAT3 phosphorylation in the hypothalamus. We reasoned that if DIO animals had both reduced ObRb expression and reduced ObRb-mediated STAT3 phosphorylation capacity, then reduced $\mathrm{ObRb}$ expression may directly contribute to the reduced responsiveness to pharmacological leptin observed in these animals. A second objective was to test whether 30 days of caloric restriction (CR) could reverse any potential deficits in ObRb expression and leptin signaling observed in DIO rats. Our final objective was to determine what effect, if any, chronically elevated leptin in the hypothalamus had on ObRb expression in lean rats.

\section{Methods and Materials}

\section{Animals}

Three-month-old male Fischer $344 \times$ Brown Norway rats were obtained from Harlan Sprague-Dawley (Indianapolis, IN, USA). Upon arrival, rats were examined and remained quarantined for 1 week. Animals were individually caged with a $12 \mathrm{~h}$ light: $12 \mathrm{~h}$ darkness cycle (0700-1900 h). Animals were cared for in accordance with the principles of the NIH Guide to the Care and Use of Experimental Animals.

\section{Experimental design 1: leptin signaling in DIO rats}

All animals were maintained on standard rat chow (Diet 2018; Harlan Teklad, Madison, WI, USA) from weaning until 2 weeks after arriving in our laboratory, at which point animals were approximately 3 months old. This CHOW diet provides $3.3 \mathrm{kcal} / \mathrm{g}$ of digestible energy and $15 \%$ of energy as fat. At this point, 65 animals were switched to a high fat/high sucrose (HF) diet (F3282; BioServ, Frenchtown, NJ, USA). This HF diet provides $5.3 \mathrm{kcal} / \mathrm{g}$ and $59 \cdot 4 \%$ of energy as fat. The top $40 \%$ of weight gainers on the HF diet were designated as DIO and the rest were removed from the study. This is similar to the designation system used previously by Levin \& Keesey (1998), who defined the top $37 \cdot 5 \%$ of weight gainers on a high energy diet as DIO. An additional group of animals were continuously maintained on CHOW to serve as the 'lean' control group. DIO and CHOW animals remained on their respective diets through to the conclusion of the study (when acute leptin sensitivity was evaluated) or until the start of CR (when remaining DIO animals were switched to a restricted $\mathrm{CHOW}$ diet). After approximately 105 days of HF feeding in DIO rats, groups of DIO and $\mathrm{CHOW}$ animals were prepared for stereotaxic surgery as described below. Animals were given a single injection into the third cerebroventricle containing $2 \mu \mathrm{g}$ leptin peptide or $4 \mu \mathrm{l}$ artificial cerebrospinal fluid (ACSF) vehicle. Sixty minutes after leptin delivery, animals were killed for the measurement of hypothalamic STAT3 phosphorylation and $\mathrm{ObRb}$ expression. This time frame was chosen because we have previously demonstrated that leptin-induced STAT3 phosphorylation peaks at $\sim 60$ min (Scarpace et al. 2001).

\section{Experimental design 2: leptin signaling following $C R$}

After more than 100 days of high-fat feeding in DIO rats, subgroups of both DIO and CHOW rats were switched to a CR diet. This CR diet provided $60 \%$ of mean, basal freely available (ad lib) caloric intake in the form of standard chow diet. Ad lib caloric intake did not differ between CHOW and DIO and averaged approximately $62.4 \mathrm{kcal} /$ day in both groups in the week prior to commencing CR. Sixty percent of this is $37 \cdot 4 \mathrm{kcal} /$ day, or $11.34 \mathrm{~g}$ of chow diet per day. Thus, approximately $11.34 \mathrm{~g}$ of chow were provided to each CR DIO and CHOW animal (DIO-CR and CHOW-CR) for 30 days. We chose to switch the DIO animals to chow diet for the CR phase of the study because of concerns about excessive ketosis and related side effects if animals were restricted on the high fat, lower carbohydrate diet. Food was placed in the stainless steel food baskets in the cage of each rat in the early evening (between 1700 and 1800 h). After 30 days of $\mathrm{CR}$, these animals were tested for acute responsiveness to $2 \mu \mathrm{g}$ i.c.v. leptin exactly as were the DIO-ad lib and CHOW-ad lib animals (see Experimental design 1).

\section{Experimental design 3: effect of central leptin overexpression on $\mathrm{ObRb}$ expression}

Animals were given a single hypothalamic injection of rAAV-leptin under the control of a tetracycline responsive element promoter (TET-Ob) or control vector encoding green fluorescent protein (GFP). The physiological responses to this TET-Ob gene therapy system, as well as details about its production and delivery, have been previously described in detail (Wilsey et al. 2002). All rats received doxycycline hydrochloride (Sigma, St Louis, MO, USA) $(400 \mu \mathrm{g} / \mathrm{ml})$ in their drinking water for 34 days, activating the leptin transgene. At this point, doxycycline was withdrawn from half of the TET-Ob-treated animals for 32 days to de-activate the leptin transgene (Ob-OFF) while half continued to receive doxycycline $(\mathrm{Ob}-\mathrm{ON})$. At this end point, animals were killed for the evaluation of hypothalamic ObRb expression as well as leptin transgene expression.

\section{Leptin administration}

Rats were anesthetized with $60 \mathrm{mg} / \mathrm{kg}$ pentobarbital and their heads were prepared for surgery. Animals were placed into a stereotaxic frame and a small incision $(1.5 \mathrm{~cm})$ was made over the midline of the skull to expose 
the landmarks of the cranium (Bregma and Lamda). The following coordinates were used for injection into the third cerebroventricle: $1.3 \mathrm{~mm}$ posterior to Bregma and $9.4 \mathrm{~mm}$ ventral from the skull surface on the midline (medial fissure), with the nose bar set at $3.3 \mathrm{~mm}$ below the ear bars (below zero) and the cannula set at $20^{\circ}$ posterior from vertical. A small hole was drilled through the skull and a 23-gauge stainless steel guide cannula was lowered to the third cerebroventricle. This was followed by an injection cannula attached to a $10 \mu \mathrm{l}$ syringe. Two micrograms of leptin dissolved in $4 \mu \mathrm{l}$ ACSF (or ACSF vehicle alone) were slowly injected (approximately $0.5 \mu \mathrm{l} / \mathrm{min}$ ) to minimize tissue damage.

\section{Vector administration}

Rats were anesthetized with $60 \mathrm{mg} / \mathrm{kg}$ pentobarbital and their heads were prepared for surgery. Animals were placed into a stereotaxic frame and a small incision $(1.5 \mathrm{~cm})$ was made over the midline of the skull to expose the landmarks of the cranium (Bregma and Lamda). The following coordinates were used for third ventricle injection: $1.8 \mathrm{~mm}$ posterior to Bregma, $1.3 \mathrm{~mm}$ anterior to Bregma, $9 \cdot 2 \mathrm{~mm}$ ventral from skull surface, on the midline (medial fissure). The nose bar was set $3.3 \mathrm{~mm}$ below zero (on same plane with ear bars) and the cannula was set $20^{\circ}$ posterior from vertical. A small hole was drilled through the skull and a 23-gauge stainless steel guide cannula was lowered to the hypothalamus. This was followed by an injection cannula attached to a $10 \mu \mathrm{l}$ syringe. We injected $5 \mu \mathrm{l}$ of viral particles in Ringer's solution at approximately $0 \cdot 25 \mu \mathrm{l} / \mathrm{min}$. Animals received either the two-vector TET-Ob system $(n=14)$ or control virus encoding GFP $(n=6)$.

\section{Tissue harvesting}

Anesthetized rats were killed by cervical dislocation. Blood was collected by cardiac puncture and serum was harvested by a 10 min centrifugation in serum separator tubes. The circulatory system was perfused with $20 \mathrm{ml}$ cold saline. Perirenal white adipose tissue (PWAT) and retroperitoneal white adipose tissue (RTWAT) and hypothalami were excised, weighed, and immediately frozen in liquid nitrogen. The hypothalamus was removed by making an incision medial to piriform lobes, caudal to the optic chiasm, and anterior to the cerebral crus to a depth of $2-3 \mathrm{~mm}$. Tissues were stored at $-80{ }^{\circ} \mathrm{C}$ until analysis.

\section{Real-time RT-PCR}

We designed primers and a Taqman probe specific for ObRb using Primer Express software, version 1.5 (PerkinElmer Applied Biosystems, Inc., Foster City, CA, USA). The sequences for the ObRb primers were for- ward primer: 5'-GGGAACCTGTGAGGATGAGTGT3', reverse primer: 5'-TTTCCACTGTTTTCACGTTG CT-3'. The fluorescent probe sequence was: 6 FAMAGAGTCAACCCTCAGTTAAATATGCAACGCTGTAMRA. Optimization experiments showed that $300 \mathrm{nM}$ of forward primer, $900 \mathrm{nM}$ of reverse primer and $50 \mathrm{nM}$ Taqman probe gave the most reproducible results and maximally efficient PCR (i.e. lowest threshold cycle $\left(\mathrm{C}_{\mathrm{T}}\right)$ values). Total RNA $(6 \mu \mathrm{g})$ was treated with RNase-free DNase using a DNA-free kit (Ambion, Inc., Austin, TX, USA). First-strand cDNA was generated from $1.6 \mu \mathrm{g}$ RNA in a $40 \mu \mathrm{l}$ volume using random primers (Gibco BRL, Gaithersburg, MD, USA) containing $200 \mathrm{U}$ of M-MLV reverse transcriptase (Gibco BRL). Real-time PCR for ObRb was performed on $100 \mathrm{ng}$ cDNA template in a $50 \mu$ total volume including Taqman RT-PCR Master Mix (Applied Biosystems, Foster City, CA, USA) using an ABI Prism GeneAmp 5700 Sequence Detection System (Applied Biosystems). ObRb expression was quantified using an $18 \mathrm{~S}$ rRNA standard (Applied Biosystems) and the $\Delta \Delta \mathrm{C}_{\mathrm{T}}$ method (Bustin 2000). Briefly, a $\Delta \mathrm{C}_{\mathrm{T}}$ value was calculated for each sample by subtracting the $\mathrm{C}_{\mathrm{T}}$ for 18S rRNA amplification from the $\mathrm{C}_{\mathrm{T}}$ for ObRb. A calibrator is then chosen and subtracted from each $\Delta \mathrm{C}_{\mathrm{T}}$ value to yield $\Delta \Delta \mathrm{C}_{\mathrm{T}}$. The mean $\Delta \mathrm{C}_{\mathrm{T}}$ in the control group (CHOW) was chosen as the calibrator for $\Delta \Delta \mathrm{C}_{\mathrm{T}}$ calculation. Finally, a $2^{-\Delta \Delta \mathrm{CT}}$ value was calculated for each sample to yield a quantitative measure of $\mathrm{ObRb}$ expression relative to $18 \mathrm{~S}$ rRNA. Units are arbitrary.

\section{STAT3/phosphorylated-STAT3 assay}

These methods were described in detail previously (Scarpace et al. 2000). Briefly, hypothalamus was sonicated in $10 \mathrm{mM}$ Tris- $\mathrm{HCl}, \mathrm{pH} 6 \cdot 8,2 \%$ SDS, and $0.08 \mu \mathrm{g} / \mathrm{ml}$ okadaic acid plus protease inhibitors (polymethylsulfonyl fluoride, benzamidine and leupeptin) (an aliquot of this sonicate was frozen for RNA analysis). Sonicate was diluted and quantified for protein using a detergentcompatible Bradford assay. Samples were boiled and separated on a $7 \cdot 5 \%$ agarose, Tris- $\mathrm{HCl}$ gel (BioRad, Hercules, CA, USA) and electrotransferred to nitrocellulose membrane. Immunoreactivity was assessed with an antibody specific to phosphorylated-STAT3 (antibody kit from New England Biolabs, Beverly, MA, USA). Immunoreactivity was visualized by chemiluminescence detection (Amersham Life Sciences, Piscataway, NJ, USA) and quantified by video densitometry (BioRad). Following phosphorylated-STAT3 quantification, membranes were stripped of antibody with Immunopure (Pierce, Rockford, IL, USA) and immunoreactivity was reassessed using a total STAT3 antibody. Since total STAT3 was not affected by dietary group or any treatment, STAT3 phosphorylation is expressed as phosphorylated-STAT3/ total STAT3 in each sample. 


\section{$\mathrm{O} b \mathrm{Rb}$ protein in hypothalamus}

$\mathrm{ObRb}$ protein was evaluated by Western analysis on the same hypothalamic tissue used for the phosphorylatedSTAT3/STAT3 assay. Samples were sonicated in a protease-inhibiting buffer as described above. Forty micrograms of protein were loaded into $15 \mu \mathrm{l}$ wells of a $5 \%$ agarose, Tris- $\mathrm{HCl}$ gel (BioRad) and then separated at $\sim 90 \mathrm{~V}$ for $70 \mathrm{~min}$. Samples were electrotransferred to a nitrocellulose membrane and then incubated with an ObRb primary antibody (Linco Research, St Louis, MO, USA). Immunoreactivity was visualized by chemiluminescence detection (Amersham Life Sciences) and quantified by video densitometry (BioRad).

\section{Leptin $m R N A$ levels in white adipose tissue}

RTWAT (300 mg/sample) was sonicated in guanidine buffer, phenol extracted, and isopropanol precipitated using a modification of the method of Chomczynski \& Sacchi (1987). Isolated RNA was resuspended in ribonuclease-free water and quantified by spectrophotometry. Integrity was verified using 1\% agarose gels stained with ethidium bromide. For dot blot analysis, multiple concentrations of RNA were immobilized on nylon membranes using a dot blot apparatus (BioRad). Membranes were baked in a UV crosslinking apparatus. Membranes were then prehybridized in $10 \mathrm{ml}$ Quickhyb (Stratagene, La Jolla, CA, USA) for 30 min followed by hybridization in the presence of a labeled probe for leptin mRNA and $100 \mu \mathrm{g}$ salmon sperm DNA. After hybridization for $2 \mathrm{~h}$ at $65{ }^{\circ} \mathrm{C}$, the membranes were washed and exposed to a phosphor imaging screen for $72 \mathrm{~h}$. The screen was then scanned using a Phosphor Imager (Molecular Dynamics, Sunnyvale, CA, USA) and analyzed by Image Quant Software (Molecular Dynamics). Data are expressed as ObRb mRNA per total RTWAT pad.

\section{Serum leptin}

Serum leptin was measured using a mouse leptin ELISA Kit from Crystal Chem. Inc. (Chicago, IL, USA) on blood harvested at killing by cardiac puncture.

\section{Statistical analysis}

All data are expressed as means \pm S.E.M. The $\alpha$ level was set at 0.05 for all analyses. Comparisons of mean food intake and body weight gain in DIO vs CHOW rats were made by a repeated-measures two-way ANOVA with time and diet as factors. Comparisons of absolute body weights of CHOW vs DIO rats were made by Student's $t$-test. Comparison in absolute body weights of $\mathrm{CHOW}-\mathrm{ad}$ lib, DIO-CR, and $\mathrm{CHOW}-\mathrm{CR}$ during the $\mathrm{CR}$ period were made by a repeated-measures two-way ANOVA with time and diet as factors. Comparisons in $\Delta$ body mass during the $\mathrm{CR}$ experiment (CHOW-ad lib, DIO-CR and $\mathrm{CHOW}-\mathrm{CR}$ groups) and $\mathrm{ObRb}$ expression in the TET-Ob experiment were made by one-way ANOVA with Tukey's post-hoc test. All other comparisons were made by two-way ANOVA with dietary group (DIO vs $\mathrm{CHOW}$ ) and treatment (leptin vs ACSF or ad lib vs CR) as factors. Note that the effects of leptin and CR on STAT3 phosphorylation in DIO and CHOW were analyzed with separate two-way ANOVAs, although the data are represented in a single graph. When only main effects were significant, relevant pairwise comparisons were made using the Bonferroni multiple comparison method with the error rate corrected for the number of contrasts (Rao 1998). When there was an interaction, factors were separated and a further one-way ANOVA was applied with a Bonferroni multiple comparison posthoc. When separation of factors resulted in only two population means to compare, the one-way ANOVA was replaced with Student's $t$-test. GraphPad Prism software version 4.0 (San Diego, CA, USA) was used for all statistical analysis and graphing. GraphPad QuickCalc (graphpad.com) was used for post-hoc analysis of two-way ANOVAs. Unless otherwise noted: ${ }^{*} P<0 \cdot 05,{ }^{* *} P<0 \cdot 01$, $* * * P<0 \cdot 001$.

\section{Results}

\section{Food intake body weight}

At the start of HF-feeding, there was no difference in body mass between DIO and CHOW rats $(286 \pm 4.4$ vs $286 \pm 6.0 \mathrm{~g}$ respectively). Through 105 days of HFfeeding, DIO animals gained $39 \cdot 3 \%$ more mass than CHOW controls (Fig. 1A), translating to $12 \%$ greater weight at this point $(467 \pm 5.2$ vs $415 \pm 8.9 \mathrm{~g}$ respectively, $P<0 \cdot 0001)$. Although there was a transient increase in caloric intake immediately after switching pre-DIO animals to the HF diet (data not shown), energy intake returned to that of $\mathrm{CHOW}$ animals within 5 days, after which point caloric intake did not differ between $\mathrm{CHOW}$ and DIO through to the conclusion of the experiment $(59 \cdot 7 \pm 1.40 \mathrm{kcal} /$ day in $\mathrm{CHOW}, 61.1 \pm 0.65 \mathrm{kcal} /$ day in DIO). This is consistent with earlier reports that DIO animals have increased feed efficiency and increased rate of adipose deposition on a high-fat diet without hyperphagia (Levin \& Dunn-Meynell 2000).

After approximately 115 days of HF-feeding in DIO rats, subgroups of DIO and $\mathrm{CHOW}$ animals were put on a CR diet, providing $60 \%$ of ad lib caloric intake. Note that the CR diet that was provided to both $\mathrm{CHOW}-\mathrm{CR}$ and DIO-CR was in the form of standard chow as described in Methods. By the end of the 30 day CR period, DIO-CR lost $62 \pm 3.5 \mathrm{~g}$, eliminating the difference in body weights between DIO-CR and CHOW-ad 
(A)

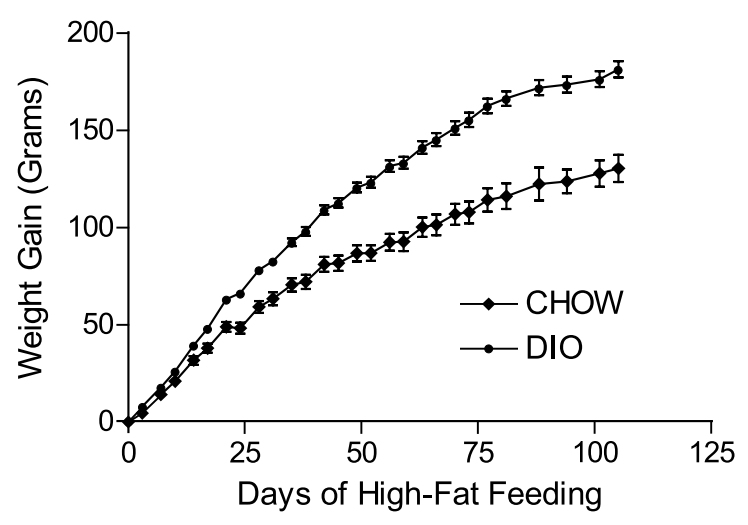

(B)

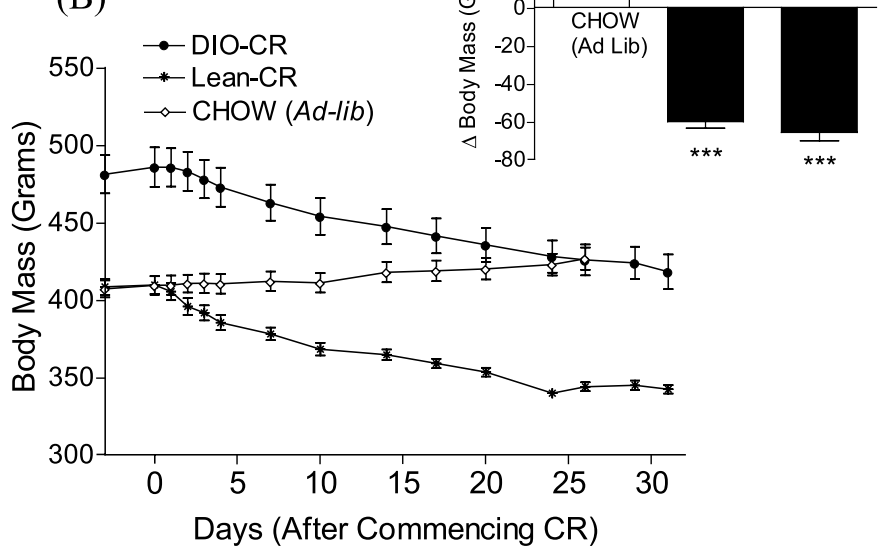

Figure 1 (A) $\Delta$ body mass during HF feeding. Values represent means \pm S.E.M. of $\mathrm{CHOW}(n=9)$ and DIO $(n=27)$ rats. By repeated-measures two-way ANOVA, significance was found for both the time $(F=1115 \cdot 8, P<0 \cdot 0001)$ and dietary group $(F=44 \cdot 39$, $P<0 \cdot 0001)$ main effects. The interaction between time and diet was also significant $(F=36 \cdot 92, P<0 \cdot 0001)$. (B) Body mass during CR. By repeated-measures two-way ANOVA, significance was found for both the time $(F=292 \cdot 96, P<0 \cdot 0001)$ and $C R(F=28 \cdot 95, P<0 \cdot 0001)$ main effects. The interaction between time and $C R$ was also significant $(F=154 \cdot 1, P<0 \cdot 0001)$. Inset: $\Delta$ body mass during the $C R$ period. By one-way ANOVA and Tukey post-hoc analysis, ${ }^{* * *} P<0.0001$ for difference in total $\Delta$ body mass between CHOW-CR and CHOW-ad lib group, ${ }^{* *} P<0 \cdot 0001$ for difference in $\Delta$ body mass between DIO-CR and CHOW-ad lib.

lib (Fig. 1B). CHOW-CR lost $65 \pm 4 \cdot 1 \mathrm{~g}$ during CR, leaving this group with $20 \cdot 4 \%$ lower mean body weights than $\mathrm{CHOW}$-ad lib controls (Fig. 1B).

Adiposity, white fat leptin expression and serum leptin

Visceral adiposity (as represented by sum of RTWAT and PWAT depots) was elevated $2 \cdot 46$-fold in DIO with respect to $\mathrm{CHOW}$ rats (Fig. 2A). Thirty days of CR reduced visceral adiposity by $55 \%$ in CHOW and by $35 \%$ in DIO (Fig. 2A). However, visceral adiposity after CR in DIO remained $60 \%$ above that of $\mathrm{CHOW}_{-a d}$ lib animals (Fig. 2A). Although $\mathrm{CHOW}$ experienced a slightly greater loss in visceral adiposity during CR when expressed as a percent of starting adiposity, the magnitude of visceral fat loss was actually greater in DIO (mean
(A)

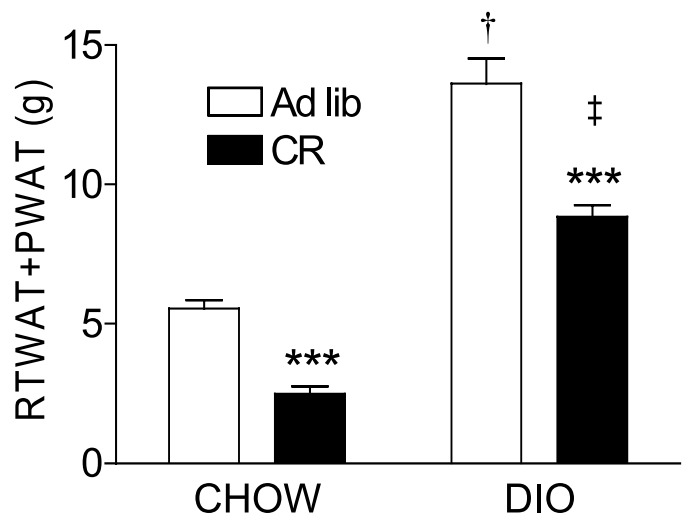

(B)

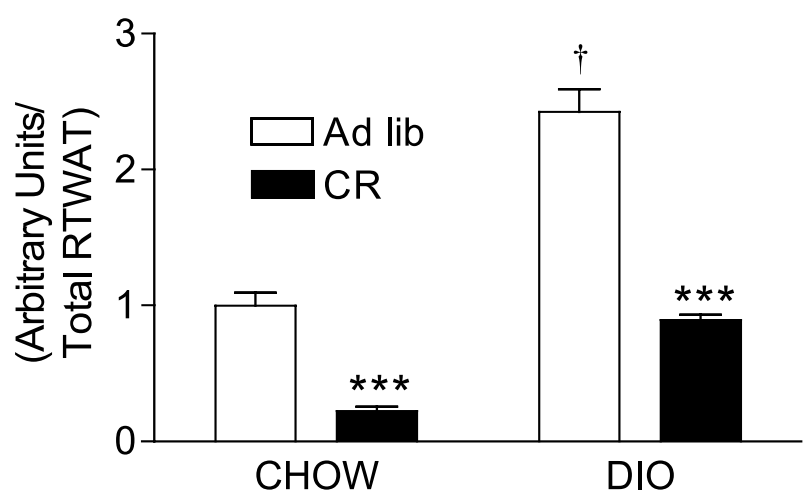

Figure 2 (A) Visceral adiposity (sum of RTWAT and PWAT) at killing. Values represent means \pm S.E.M. of $\mathrm{CHOW}$-ad lib $(n=8)$, DIO-ad lib $(n=5)$, CHOW-CR $(n=8)$, and DIO-CR $(n=5)$. By two-way ANOVA, significance was found for both the CR $(\mathrm{F}=79 \cdot 01, P<0 \cdot 0001)$ and dietary group $(\mathrm{F}=267 \cdot 42, P<0 \cdot 0001)$ main effects. By post-hoc analysis, adiposity was significantly greater in $\mathrm{DIO}$ compared with $\mathrm{CHOW}$ rats $(\dagger P<0 \cdot 001)$. By post-hoc analysis, effect of $\mathrm{CR}$ was significant in both $\mathrm{CHOW}$ $\left({ }^{* *} P<0 \cdot 001\right)$ and DIO (***P<0.001) rats. Visceral adiposity in DIO-CR remained above that of CHOW-ad lib $(\ddagger P<0 \cdot 001)$. (B) Leptin expression in RTWAT at killing. By two-way ANOVA, both diet and CR main effects were significant $(F=126 \cdot 75$, $P<0.0001 ; F=153.79, P<0.0001$ respectively), as was the interaction between the main effects $(F=16.54, P<0.001)$. By post-hoc analysis, $+P<0.0001$ for difference in basal RTWAT leptin expression in CHOW-ad lib and DIO-ad lib; ${ }^{* * *} P<0 \cdot 0001$ for effect of $\mathrm{CR}$ in both CHOW and DIO. 


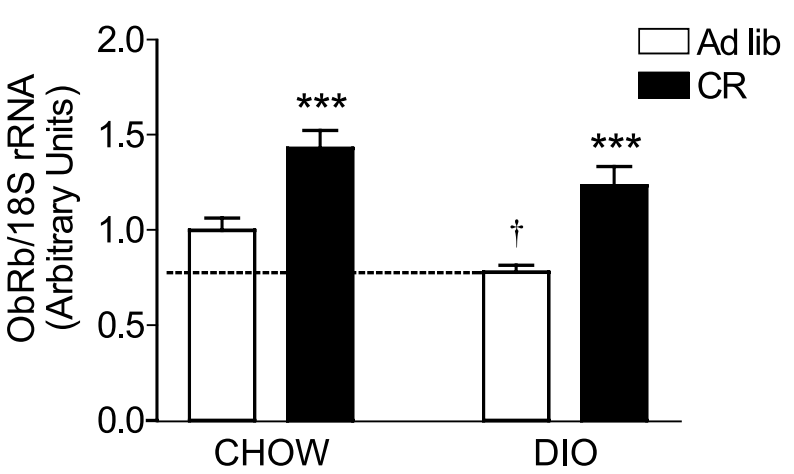

Figure $3 \mathrm{ObRb}$ expression in the hypothalamus. Values represent means \pm S.E.M. of CHOW-ad lib $(n=8)$, DIO-ad lib $(n=17)$, CHOW-CR $(n=8)$, and DIO-CR $(n=5)$. By two-way ANOVA, significance was found for both the $C R(F=39 \cdot 85, P<0 \cdot 0001)$ and dietary group $(F=8.81, P<0.01)$ main effects. By post-hoc analysis, basal ObRb expression was significantly reduced in DIO-ad lib vs $\mathrm{CHOW}$-ad lib rats, $+P<0 \cdot 05$. Effect of $\mathrm{CR}$ was significant in both CHOW $\left({ }^{* * *} P<0 \cdot 001\right)$ and DIO $\left({ }^{* * *} P<0 \cdot 001\right)$ rats.

visceral fat loss was $3.05 \mathrm{~g}$ in $\mathrm{CHOW}$ and $4.79 \mathrm{~g}$ in DIO). Leptin expression per RTWAT pad was elevated nearly 2.5-fold in DIO-ad lib compared with CHOW-ad lib (Fig. 2B). Thirty days of CR caused a $78 \%$ decrease in RTWAT leptin expression in CHOW animals and a $63 \%$ decrease in DIO (Fig. 2B). The effect of CR in DIO rats brought RTWAT leptin expression to a level comparable with that in $\mathrm{CHOW}$-ad lib (Fig. 2B). Consistent with the adiposity data, serum leptin was elevated approximately $2 \cdot 4$-fold in DIO animals compared with CHOW $(17 \cdot 2 \pm 1 \cdot 37$ and $7 \cdot 3 \pm 0.53 \mathrm{ng} / \mathrm{ml}$ respectively, $P<0 \cdot 0001)$. An accurate measure of endogenous serum leptin in both $\mathrm{CR}$ groups (CHOW-CR and DIO-CR) could not be obtained due to leakage of the supramaximal leptin injection from the cerebrospinal fluid.

\section{$\mathrm{ObRb}$ expression and protein levels in the hypothalamus}

DIO animals had a $22 \%$ reduction in ObRb expression in the hypothalamus with respect to $\mathrm{CHOW}$ rats (Fig. 3). $\mathrm{CR}$ resulted in a $43 \%$ increase in ObRb expression in CHOW-CR vs CHOW and a $58 \%$ increase in DIO-CR vs DIO (Fig. 3). The $\mathrm{C}_{\mathrm{T}}$ value for the $18 \mathrm{~S}$ rRNA control signal was not affected by obesity or $\mathrm{CR}\left(\mathrm{C}_{\mathrm{T}}\right.$ values for $18 \mathrm{~S}$ rRNA were $16 \cdot 60 \pm 0 \cdot 11$ in CHOW-ad lib, $16 \cdot 43 \pm 0 \cdot 15$ in DIO-ad lib, $16.78 \pm 0.11$ in CHOW-CR, and $16.79 \pm 0.14$ in DIO-CR), suggesting that the observed changes in $\mathrm{ObRb}$ where due to changes in the absolute level of ObRb mRNA.

Changes in $\mathrm{ObRb}$ protein qualitatively mirrored the mRNA data. DIO animals had a 30\% reduction in $\mathrm{ObRb}$ protein with respect to $\mathrm{CHOW}$ (Fig. 4). CR caused modest $(\sim 20 \%)$, but statistically significant increases in

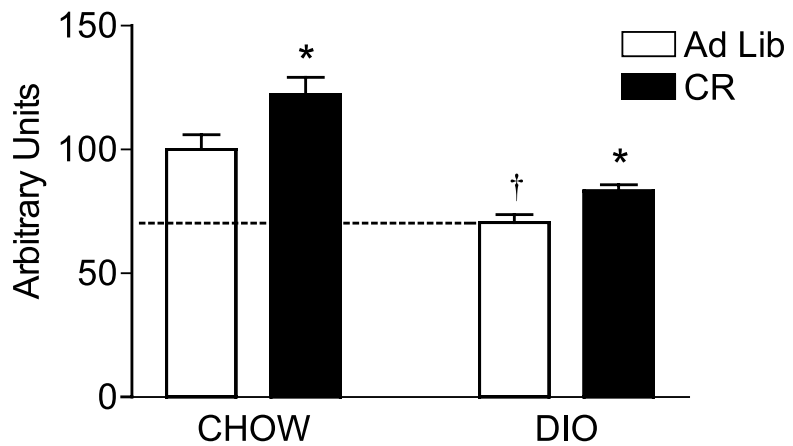

Figure $4 \mathrm{ObRb}$ protein in the hypothalamus. Values represent means \pm S.E.M. of CHOW-ad lib $(n=8)$, DIO-ad lib $(n=17)$, CHOW-CR $(n=8)$, and DIO-CR $(n=5)$. By two-way ANOVA, significance was found for both the $C R(F=8.93, P<0.01)$ and dietary group $(F=34 \cdot 60, P<0 \cdot 0001)$ main effects. By post-hoc analysis, basal ObRb protein was significantly reduced in DIO-ad lib vs $\mathrm{CHOW}$-ad lib, $+P<0 \cdot 05$. Effect of $\mathrm{CR}$ was significant in both CHOW $\left({ }^{*} P<0.05\right)$ and DIO $\left({ }^{*} P<0.05\right)$ animals.

$\mathrm{ObRb}$ protein in both $\mathrm{CHOW}-\mathrm{CR}$ and $\mathrm{DIO}-\mathrm{CR}$ rats (Fig. 4).

\section{Hypothalamic STAT3 phosphorylation}

Basal (unstimulated) STAT3 phosphorylation was elevated approximately 3 -fold in DIO compared with $\mathrm{CHOW}$ rats (Fig. 5). However, we suspected that this high-fat feedinginduced stimulation does not represent maximal STAT3 phosphorylation. Thus, we administered a central dose of leptin that would cause maximal leptin-induced STAT3 phosphorylation at $60 \mathrm{~min}$. The dose of leptin used in this study $(2 \mu \mathrm{g})$ is termed 'supramaximal leptin' because it is $>8$ times the i.c.v. dose required to achieve maximal STAT3 phosphorylation in both lean and obese F334 $\times$ BN rats in our laboratory (Scarpace et al. 2001). A single i.c.v. injection containing supramaximal leptin caused a $6 \cdot 1$-fold increase in STAT3 phosphorylation in CHOW rats, yet caused only a $1 \cdot 7$-fold induction in STAT3 phosphorylation in DIO rats (Fig. 5). However, basal levels of STAT3 phosphorylation were elevated 3-fold in DIO vs CHOW animals. Nevertheless, there was still a small but significant $16 \%$ decrease in maximally stimulated STAT3 phosphorylation capacity in DIO vs CHOW rats $(P<0 \cdot 05$, Fig. 5$)$. Maximal STAT3 activation was then examined after 30 days of CR. CR caused a $45 \%$ increase in maximal leptin-induced hypothalamic STAT3 phosphorylation capacity in CHOW rats (i.e. $\mathrm{CHOW}-\mathrm{CR}$ vs $\mathrm{CHOW}-\mathrm{ad}$ lib) and a dramatic $85 \%$ increase in DIO (DIO-CR vs DIO-ad lib), completely reversing the obesity-associated impairment in signaling capacity (Fig. 5). Similar to the pattern observed with $\mathrm{ObRb}$ expression, the obesity-associated deficit was completely reversed as DIO-CR rats displayed 56\% greater leptin-induced STAT3 phosphorylation capacity 
(A)

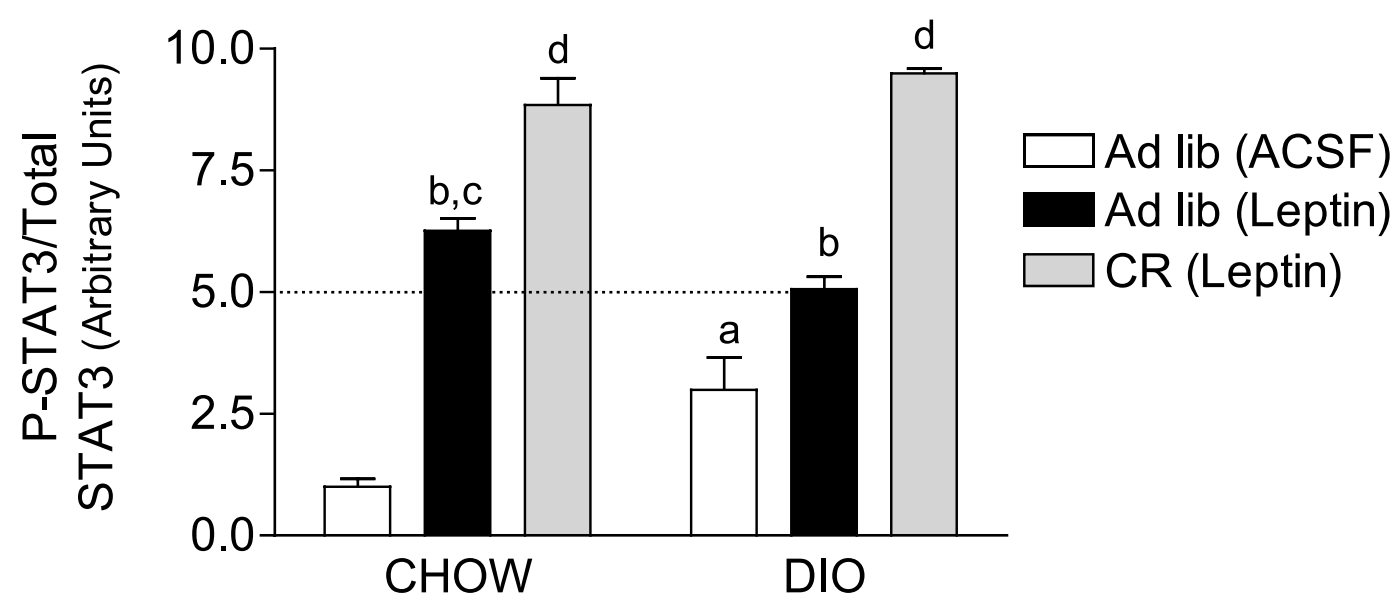

(B)

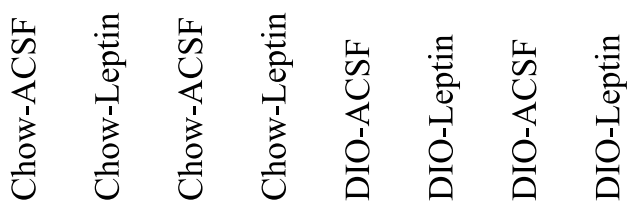

P-STAT3

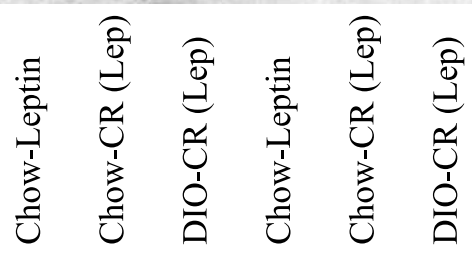

\section{P-STAT3}

Figure 5 (A) STAT3 phosphorylation $1 \mathrm{~h}$ after i.c.v. leptin $(2 \mu \mathrm{g})$ or ACSF administration in ad lib-fed animals; STAT3 phosphorylation $1 \mathrm{~h}$ after i.c.v. leptin in CR animals. Values represent means \pm S.E.M. of CHOW-ad lib, ACSF) $(n=5)$, CHOW-ad lib, leptin) ( $n=6)$, CHOW-CR (leptin) $(n=8)$, DIO-ad lib, ACSF) $(n=6)$, DIO-ad lib, leptin) $(n=6)$, DIO-CR (leptin) $(n=5)$. By two-way ANOVA with dietary group and leptin as factors, leptin main effect was significant $(F=84 \cdot 40, P<0 \cdot 0001)$, as was interaction between dietary group and leptin $(F=14 \cdot 1, P<0 \cdot 001)$, but diet main effect was not significant $(F=1 \cdot 75)$. By a second two-way ANOVA with diet and $C R$ as factors, significance was found only for the $C R$ main effect $(F=60 \cdot 75, P<0 \cdot 0001)$. By post-hoc analysis, (a) $P<0 \cdot 05$ for difference in basal STAT3 phosphorylation in CHOW vs DIO (white bars); (b) $P<0.0001$ for effect of leptin in $\mathrm{CHOW}$ and $P<0.01$ for effect of leptin in DIO (solid bars vs white bars); (c) $P<0.05$ for difference in maximally stimulated STAT3 phosphorylation in CHOW-Lep and DIO-Lep (gray bars); (d) $P<0.001$ and $P<0.001$ respectively, for effect of CR on STAT3 phosphorylation capacity in CHOW and DIO (gray vs solid bars). (B) Representative gels of phosphorylated-STAT3 (P-STAT3) in the hypothalamus. Top, first four lanes demonstrate effect of leptin in CHOW rats. Top, last four lanes demonstrate leptin effect in DIO rats. Bottom gel section compares maximal leptin-induced P-STAT3 in CHOW and CHOW-CR and DIO-CR.

compared with the leaner CHOW-ad lib animals (Fig. 5). Total STAT3 in the hypothalamus was not affected by diet or acute leptin (CHOW-ACSF, 1·00 $\pm 0 \cdot 11$ arbitrary units; CHOW-Lep, 1.02 $\pm 0 \cdot 06$; CHOW-CR (Lep), $0.95 \pm 0.03$; DIO-ACSF, $1.03 \pm 0 \cdot 07$; DIO-Lep, $1.08 \pm$ 0.04; DIO-CR, $0 \cdot 97 \pm 0 \cdot 06$ ). 


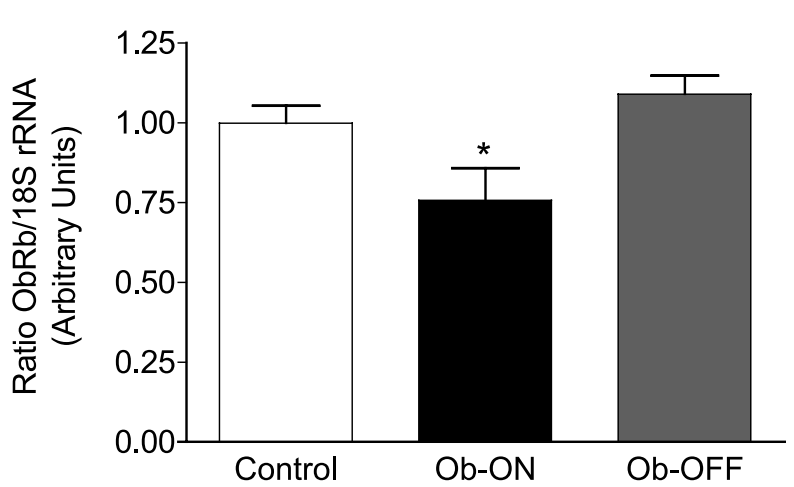

Figure 6 Effect of leptin transgene on ObRb expression in the hypothalamus. Values represent means \pm S.E.M. of control $(n=5)$, Ob-ON $(n=4)$ and Ob-OFF $(n=4)$. Overall significance was detected by one-way ANOVA ( $F=5 \cdot 494, P<0 \cdot 05)$. By Tukey post-hoc analysis, only the difference between $\mathrm{Ob}-\mathrm{ON}$ and Ob-OFF was significant $\left({ }^{*} P<0 \cdot 05\right)$.

\section{Interaction of hypothalamic leptin transgene and $\mathrm{ObRb}$ expression}

To further explore the role of elevated central leptin in regulating hypothalamic $\mathrm{ObRb}$ expression, we administered rAAV-leptin under the control of a tetracyclineresponsive promoter (TET-Ob) to a subset of normal, $\mathrm{CHOW}$ animals. This TET-Ob system allows us to regulate leptin transgene expression via doxycycline in the drinking water (Wilsey et al. 2002). Animals that had their leptin transgene continuously activated for 2 months (TET-Ob-ON) tended to have reduced hypothalamic $\mathrm{ObRb}$ expression compared with rats administered a control vector expressing GFP (Fig. 6). Moreover, a subgroup of TET-Ob-treated animals that had their leptin transgene activated for 1 month and then silenced for 1 month had significantly greater hypothalamic ObRb expression compared with the TET-Ob-ON group (Fig. 6). Our ability to activate and silence the leptin transgene in the hypothalamus was confirmed by RT-PCR (Wilsey et al. 2002).

\section{Discussion}

We previously demonstrated that the DIO F344 $\times$ BN model is non-responsive to the normally potent anorectic and lipopenic effects of hypothalamic leptin overexpression, and that these DIO animals also have reduced ObRb expression in the hypothalamus (Wilsey et al. 2003). The major objective of the present study was to determine if the physiological leptin resistance and reduced ObRb expression in this DIO model is associated with deficits in leptin signal transduction capacity. More specifically, we wanted to test the hypothesis that DIO animals have a lower ceiling of leptin-induced hypothalamic STAT3 phosphorylation. Our second objective was to determine if such a deficit in leptin signaling could be reversed by $\mathrm{CR}$.

Here, we report that DIO animals have a significant reduction in hypothalamic ObRb expression ( $-22 \%)$ and protein $(-30 \%)$ coupled with a 3 -fold elevation in basal STAT3 phosphorylation. While we suspect that the elevation in basal STAT3 phosphorylation in DIO rats is a product of their hyperleptinemia, it is possible that one or more other cytokines contribute to this phenomenon. Nonetheless, when fully stimulated by leptin, maximal STAT3 phosphorylation capacity in DIO rats is diminished by $16 \%$ compared with $\mathrm{CHOW}$ rats. Moreover, we observed a 58\% increase in hypothalamic ObRb expression, an 18\% increase in $\mathrm{ObRb}$ protein, and an impressive $85 \%$ increase in maximal leptin-induced STAT3 phosphorylation capacity in DIO rats following 30 days of CR. CR caused similar increases in hypothalamic ObRb expression and STAT3 phosphorylation capacity in lean CHOW animals.

Changes in $\mathrm{ObRb}$ expression, including the decrease in DIO rats and increase with CR, were accompanied by qualitatively similar changes in STAT3 phosphorylation capacity. The significance of this relationship has yet to be established. It has been well documented that the ObRb catalyzes STAT3 phosphorylation via receptor-bound JAK2, the latter of which docks on the intracellular domain of $\mathrm{ObRb}$ homodimers upon leptin activation of the receptor complex (Sweeney 2002). We believe that a decrease in $\mathrm{ObRb}$ expression and receptor number may decrease the available amount of $\mathrm{ObR} b$ for JAK2 binding and activation. This, in turn, may decrease maximal leptin-induced STAT3 phosphorylation capacity. Since ObRb protein and leptin-induced STAT3 phosphorylation capacity were reduced by a similar magnitude in DIO rats, we do not believe that the coupling of the ObRb to JAK2-STAT3 signaling is impaired in obesity. However, coupling of the ObRb to STAT3 signaling may be improved by $\mathrm{CR}$ as signaling capacity is increased to a much greater extent than $\mathrm{ObRb}$ protein following CR. This pattern of a disproportionate increase in leptin signaling capacity subsequent to $\mathrm{CR}$ is especially evident in DIO rats. DIO-CR rats nearly doubled their ceiling of leptin-induced STAT3 phosphorylation despite the relatively meager induction of $\mathrm{ObR} b$.

The mechanism behind reduced $\mathrm{ObRb}$ expression in $\mathrm{DIO}$ animals and enhanced $\mathrm{ObRb}$ expression following $\mathrm{CR}$ remains in question. However, it may involve the ability of leptin itself to regulate $\mathrm{ObR} b$ expression. Martin et al. (2000) reported a decrease in both ObRb expression and protein following 28 days of peripheral leptin infusion. Moreover, Fernandez-Galaz et al. (2002) showed that approximately 1 month of food restriction (leading to a $40 \%$ reduction in serum leptin) reverses the reduction in $\mathrm{ObR} b$ expression observed in hyperleptinemic aged-obese Wistar rats. However, neither of these studies attempted to differentiate between the effects of peripheral and central 
leptin. To further explore the ability of leptin to regulate $\mathrm{ObRb}$ expression, we overexpressed leptin in the hypothalami of a separate group of normal CHOW animals. Consistent with negative regulation of hypothalamic $\mathrm{ObRb}$ expression by leptin, ObRb expression was reduced by $\sim 25 \%$ after 2 months of continuous transgene activation compared with animals treated with a control vector (Ob-ON vs control). More impressively, ObRb expression was $\sim 44 \%$ greater in a group of animals in which we silenced the leptin transgene for 1 month following a month of activation (Ob-OFF) compared with the group with the continuously activated leptin transgene $(\mathrm{Ob}-\mathrm{ON})$. The $\mathrm{Ob}-\mathrm{ON}$ animals, which had high central leptin expression and presumably high leptin levels at the level of the hypothalamic receptors, had $\sim 70-80 \%$ less serum leptin than Ob-OFF and controls (Wilsey et al. 2002). This shows that the decrease in ObRb expression in $\mathrm{Ob}-\mathrm{ON}$ was a specific effect of central leptin as opposed to an indirect effect of peripheral hyperleptinemia.

Short- and long-term CR has been shown to dramatically reduce serum leptin, usually to a much greater extent than would be predicted based on the loss of adiposity (Shimokawa \& Higami 2001, Miyawaki et al. 2002). In the present study, we report significant reductions in leptin expression in white fat following 30 days of CR $(-78 \%$ in $\mathrm{CHOW}$ and $-63 \%$ in DIO rats). Although we were unable to obtain a reliable measure of endogenous serum leptin in our CR rats, the diminished leptin expression in the white fat of CR animals is consistent with reduced serum leptin following food restriction. Taken together, the changes in peripheral leptin expression following high-fat feeding and CR along with the TET-Ob data discussed in the previous paragraph support our hypothesis that leptin is regulating hypothalamic ObRb expression, and this is a direct effect of leptin in the central nervous system.

We previously demonstrated a reduction in both leptininduced hypothalamic STAT3 phosphorylation capacity and $\mathrm{ObRb}$ protein in aged-obese $\mathrm{F} 344 \times \mathrm{BN}$ male rats (Scarpace et al. 2001). Fernandez-Galaz et al. (2002) reported both reduced $\mathrm{ObRb}$ expression and receptor number in the hypothalami of aged-obese Wistar rats, as well as blunted physiological leptin responsiveness. These deficits in aged-obese Wistar rats were completely reversed by 3 months of moderate ( $\sim 80 \%$ ad lib) CR (Fernandez-Galaz et al. 2002). Our present data suggest that similar defects in $\mathrm{ObRb}$ expression are present in young adult DIO rats and, moreover, that ObRb expression and STAT3 phosphorylation capacity can be dramatically increased in both DIO animals and their $\mathrm{CHOW}$ lean counterparts by 30 days of CR. It seems plausible that a similar mechanism may be involved in the changes in $\mathrm{ObRb}$ expression and leptin sensitivity observed in both aged-obese and DIO animals. Namely, the reductions in $\mathrm{ObRb}$ expression and signaling capacity in both aged and DIO animals may be due to chronic hyperleptinemia, and, similarly, the restoration of $\mathrm{ObRb}$ expression and leptin sensitivity following $\mathrm{CR}$ in aged and DIO animals may be secondary to reduced leptin levels.

It seems unlikely that the modest 30\% decrease in $\mathrm{ObRb}$ protein and slight attenuation in signaling capacity through the JAK-STAT cascade in DIO animals are the sole contributors to the complete physiological nonresponsiveness to central leptin overexpression we previously reported in these animals (Wilsey et al. 2003). Rather, these data are suggestive of either downstream or parallel contributors to leptin resistance. As an example of a possible downstream contributor, the ability of leptin to stimulate the anorexic/thermogenic melanocortin system may be impaired in obesity. Consistent with this, Hansen et al. (2001) demonstrated that DIO Sprague-Dawley rats have reduced endogenous $\alpha$-melanocyte-stimulating hormone levels in the paraventricular nucleus and are hyperresponsive to the acute anorectic effects of i.c.v. injections of the peptide. It is also possible that signal transduction through parallel cascades is impaired in DIO animals. It has been recently demonstrated that the ObRb can signal through an insulin receptor substratephosphatidylinositol 3-kinase-phosphatidyl inositol trisphosphate cascade (Kim et al. 2000, Zhao et al. 2002, Niswender et al. 2003).

Regardless of the part played by parallel or downstream pathways, signaling through STAT3 appears to be an absolute requirement for leptin's effects on energy balance (Bates et al. 2003). As such, the potential role of even small changes in ObRb-STAT3 signaling capacity in the etiology of leptin resistance should not be ignored. Moreover, it remains a possibility that changes in ObRb expression and signaling capacity in specific nuclei of the hypothalamus most critical to the regulation of energy balance were greater than we observed by analyzing the whole hypothalamus. Additional studies are necessary to determine the effects of obesity and CR on leptin signal transduction in the arcuate and other hypothalamic nuclei.

In conclusion, diminished $\mathrm{ObRb}$ expression in $\mathrm{DIO}$ rats is associated with attenuated maximal leptin-induced STAT3 phosphorylation capacity. CR reverses these deficits, increasing both $\mathrm{ObRb}$ expression and signaling capacity in DIO animals to levels at or above those observed in the highly leptin-responsive CHOW animals. Furthermore, hypothalamic overexpression of leptin causes a reversible reduction in ObRb mRNA. This demonstrates that local leptin levels negatively regulate $\mathrm{ObRb}$ expression in the hypothalamus, and suggests that this may be one of the mechanisms behind the observed changes in $\mathrm{ObR} b$ expression and signaling with obesity and following food restriction. Thus, short-term CR may be a viable strategy to restore ObRb number, signaling capacity, and, ergo, leptin sensitivity in previously leptin-resistant models of acquired obesity. 


\section{Funding}

This work was supported by the Medical Research Service of the Department of Veterans Affairs and NIH Grant AG-17047.

\section{References}

Banks WA, DiPalma CR \& Farrell CL 1999 Impaired transport of leptin across the blood-brain barrier in obesity. Peptides $\mathbf{2 0}$ 1341-1345.

Bates SH, Stearns WH, Dundon TA, Schubert M, Tso AW, Wang Y, Banks AS, Lavery HJ, Haq AK, Maratos-Flier E et al. 2003 STAT3 signalling is required for leptin regulation of energy balance but not reproduction. Nature $421856-859$.

Bustin SA 2000 Absolute quantification of mRNA using real-time reverse transcription polymerase chain reaction assays. Journal of Molecular Endocrinology 25 169-193.

Chomczynski P \& Sacchi N 1987 Single-step method of RNA isolation by acid guanidinium thiocyanate-phenol-chloroform extraction. Analytical Biochemistry 162 156-159.

El-Haschimi K, Pierroz DD, Hileman SM, Bjorbaek C \& Flier JS 2000 Two defects contribute to hypothalamic leptin resistance in mice with diet-induced obesity. Journal of Clinical Investigation 105 1827-1832.

Fernandez-Galaz C, Fernandez-Agullo T, Perez C, Peralta S, Arribas C, Andres A, Carrascosa JM \& Ros M 2002 Long-term food restriction prevents ageing-associated central leptin resistance in Wistar rats. Diabetologia 45 997-1003.

Gura T 1999 Obesity research. Leptin not impressive in clinical trial. Science 286 881-882.

Halaas JL, Boozer C, Blair-West J, Fidahusein N, Denton DA \& Friedman JM 1997 Physiological response to long-term peripheral and central leptin infusion in lean and obese mice. PNAS 94 8878-8883.

Hansen MJ, Ball MJ \& Morris MJ 2001 Enhanced inhibitory feeding response to alpha-melanocyte stimulating hormone in the diet-induced obese rat. Brain Research 892 130-137.

Kim YB, Uotani S, Pierroz DD, Flier JS \& Kahn BB 2000 In vivo administration of leptin activates signal transduction directly in insulin-sensitive tissues: overlapping but distinct pathways from insulin. Endocrinology 141 2328-2339.

Levin BE \& Keesey RE 1998 Defense of differing body weight set points in diet-induced obese and resistant rats. American Journal of Physiology. Regulatory, Integrative, and Comparative Physiology 274 R412-R419.

Levin BE \& Dunn-Meynell AA 2000 Defense of body weight against chronic caloric restriction in obesity-prone and -resistant rats. American Journal of Physiology. Regulatory, Integrative, and Comparative Physiology 278 R231-R237.

Levin BE \& Dunn-Meynell AA 2002 Reduced central leptin sensitivity in rats with diet-induced obesity. American Journal of Physiology. Regulatory, Integrative, and Comparative Physiology 283 R941-R948.

Lin S, Storlien LH \& Huang XF 2000 Leptin receptor, NPY, POMC mRNA expression in the diet-induced obese mouse brain. Brain Research 875 89-95

Madiehe AM, Schaffhauser AO, Braymer DH, Bray GA \& York DA 2000 Differential expression of leptin receptor in high- and low-fat-fed Osborne-Mendel and S5B/P1 rats. Obesity Research 8 467-474.

Martin RL, Perez E, He YJ, Dawson R Jr \& Millard WJ 2000 Leptin resistance is associated with hypothalamic leptin receptor mRNA and protein downregulation. Metabolism 49 1479-1484.

Miyawaki T, Masuzaki H, Ogawa Y, Hosoda K, Nishimura H, Azuma N, Sugawara A, Masuda I, Murata M, Matsuo T et al. 2002 Clinical implications of leptin and its potential humoral regulators in long-term low-calorie diet therapy for obese humans. European Journal of Clinical Nutrition 56 593-600.

Niswender KD, Morrison CD, Clegg DJ, Olson R, Baskin DG, Myers MG Jr, Seeley RJ \& Schwartz MW 2003 Insulin activation of phosphatidylinositol 3-kinase in the hypothalamic arcuate nucleus: a key mediator of insulin-induced anorexia. Diabetes $\mathbf{5 2}$ 227-231.

Rao PV 1998 Statistical Research Methods in the Life Sciences. Pacific Grove, CA: Brooks/Cole Publishing Company.

Scarpace PJ \& Tumer N 2001 Peripheral and hypothalamic leptin resistance with age-related obesity. Physiology and Behavior $\mathbf{7 4}$ 721-727.

Scarpace PJ, Matheny M \& Shek EW 2000 Impaired leptin signal transduction with age-related obesity. Neuropharmacology 39 1872-1879.

Scarpace PJ, Matheny M \& Tumer N 2001 Hypothalamic leptin resistance is associated with impaired leptin signal transduction in aged obese rats. Neuroscience 104 1111-1117.

Scarpace PJ, Matheny M, Zhang Y, Tumer N, Frase CD, Shek EW, Hong B, Prima V \& Zolotukhin S 2002 Central leptin gene delivery evokes persistent leptin signal transduction in young and aged-obese rats but physiological responses become attenuated over time in aged-obese rats. Neuropharmacology 42 548-561.

Scarpace PJ, Matheny M, Zolotukhin S, Tumer N \& Zhang Y 2003 Leptin-induced leptin resistant rats exhibit enhanced responses to the melanocortin agonist MTII. Neuropharmacology 45 211-219.

Shek EW \& Scarpace PJ 2000 Resistance to the anorexic and thermogenic effects of centrally administrated leptin in obese aged rats. Regulatory Peptides 92 65-71.

Shimokawa I \& Higami Y 2001 Leptin and anti-aging action of caloric restriction. Journal of Nutrition, Health, and Aging 5 43-48.

Sweeney G 2002 Leptin signalling. Cellular Signalling 14 655-663.

Van Heek M, Compton DS, France CF, Tedesco RP, Fawzi AB, Graziano MP, Sybertz EJ, Strader CD \& Davis HR Jr 1997 Diet-induced obese mice develop peripheral, but not central, resistance to leptin. Journal of Clinical Investigation 99 385-390.

Wilsey J, Zolotukhin S, Prima V, Shek EW, Matheny MK \& Scarpace PJ 2002 Hypothalamic delivery of doxycycline-inducible leptin gene allows for reversible transgene expression and physiological responses. Gene Therapy 9 1492-1499.

Wilsey J, Zolotukhin S, Prima V \& Scarpace PJ 2003 Central leptin gene therapy fails to overcome leptin resistance associated with diet-induced obesity. American Journal of Physiology. Regulatory, Integrative, and Comparative Physiology 285 R1011-R1020.

Zhao AZ, Huan JN, Gupta S, Pal R \& Sahu A 2002 A phosphatidylinositol 3-kinase phosphodiesterase 3B-cyclic AMP pathway in hypothalamic action of leptin on feeding. Nature Neuroscience $\mathbf{5} 727-728$.

Received 13 January 2004

Accepted 27 January 2004

Made available online as an

Accepted Preprint 4 February 2004 